\title{
Conformational analysis of methyl $\beta$-cellobioside by ROESY NMR spectroscopy and MD simulations in combination with the CROSREL method
}

\author{
Loes M.J. Kroon-Batenburg ${ }^{a, *}$, Jan Kroon ${ }^{a}$, Bas R. Leeflang ${ }^{b}$ \\ and Johannes F.G. Vliegenthart ${ }^{\mathrm{b}}$ \\ a Department of Crystal and Structural Chemistry, Bijvoet Center, Utrecht University, P.O. Box 80.075, \\ NL-3508 TB Utrecht (Netherlands) \\ ${ }^{b}$ Department of Bio-organic Chemistry, Bijvoet Center, Utrecht University, P.O. Box 80.075, \\ NL-3508 TB Utrecht (Netherlands)
}

(Received August 14th, 1992; accepted January 5th, 1993)

\begin{abstract}
Methyl $\beta$-cellobioside has been studied extensively by molecular dynamics (MD) simulations in water and by ROESY NMR spectroscopy in order to establish its solution structure. The MD simulations were started with four significantly different minimal energy conformations. The MD trajectories were analysed with respect to interproton distances and mobility, in order to find models for application in the analysis of NMR data. The ROESY spectra were analysed by using the CROSREL method, which allows quantitative analysis of ROESY spectra through correction for the offset dependence and incorporation of HOHAHA transfer estimates. These results were compared with data obtained from an initial rate analysis of the ROESY data and with the MD data. It is concluded that methyl $\beta$-cellobioside in aqueous solution is in the same extended conformation that is also found in the solid state.
\end{abstract}

\section{INTRODUCTION}

The spatial structure and the crystal packing of cellulose, including the orientation of the chains, has been the subject of many studies. The parallel orientation of the chains in native cellulose ${ }^{1}$ seems to be widely accepted. The chain orientation, however, in cellulose II (regenerated cellulose) is not agreed upon ${ }^{2}$. Packing analysis in combination with X-ray fibre diffraction suggests an antiparallel orientation $^{3}$, which implies a reorientation of the cellulose chains during the merceration process. Since cellulose does not really dissolve during the conversion, it is difficult to imagine the actual physical process. It has been suggested that the cellulose chains manage to fold back, in order to achieve an antiparallel orienta-

\footnotetext{
* Corresponding author.
} 


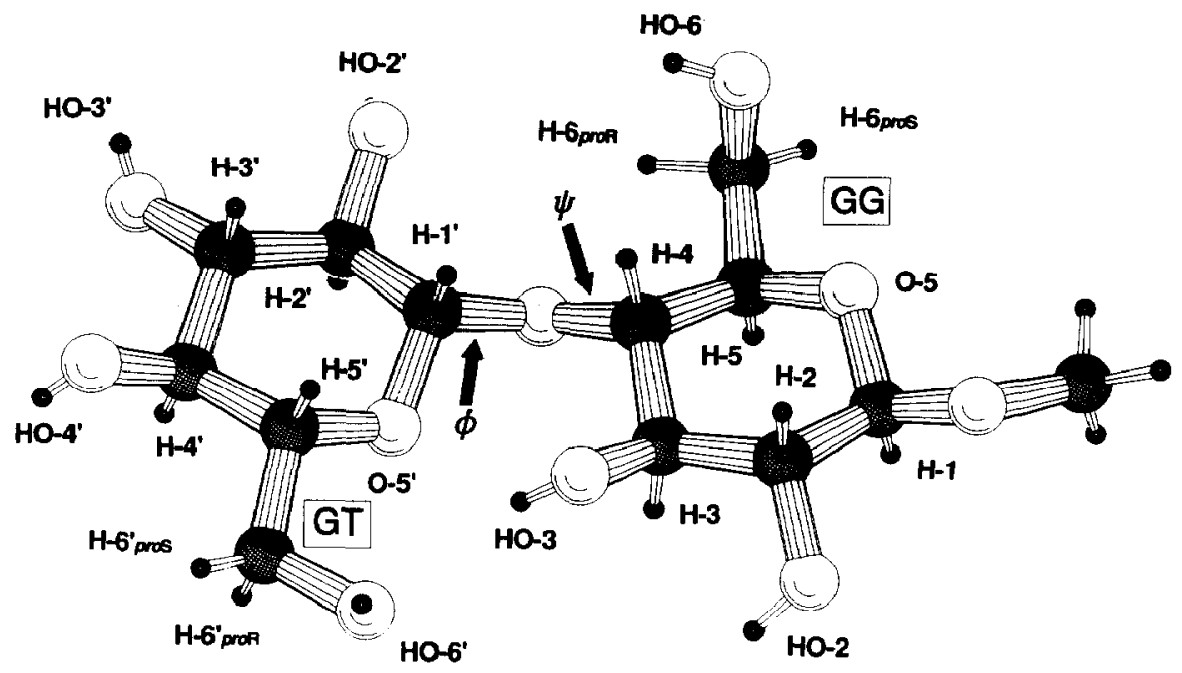

Fig. 1. Molecular model of methyl $\beta$-cellobioside. Atom labels and relevant torsional angles have been indicated. The conformation of the hydroxymethyl group is defined by a two-character code (GG, GI, and TG), indicating the orientation of the torsional angles O-6-C-6-C-5-O-5 and O-6-C-6-C-5-C-4 $(\omega)$, respectively ( $G$ means a gauche and $T$ a trans orientation). The populations of these conformations arc designated $g g, g t$, and $t g$, respectively.

tion $^{4}$. This mechanism requires the cellulose chain to adopt a folded conformation, whereas it is usually considered to be in an extended conformation. Molecular mechanics studies have indicated the presence of four energy minimum conformations for the $\beta$ - $(1 \rightarrow 4)$ linkage of two glucose residues ${ }^{5}$. In this study, the presence of these conformations has been investigated in aqueous solution by NMR spectroscopy and molecular dynamics (MD) simulations. Methyl $\beta$-cellobioside has been used as a model and is displayed in Fig. 1.

2D Nuclear Overhauser enhancement spectroscopy (NOESY) is known for its ability to give reliable estimates of interproton distances in large molecules. However, when the product of the Larmor frequency $\omega$, and the rotational correlation time $\tau_{\mathrm{c}}$ is close to $(\sqrt{5}) / 2$, as is the case for smaller molecules, no NOE can be measured. Bothner-By et al. $^{6}$ introduced rotating frame nuclear Overhauser enhancement spectroscopy (ROESY), which they called CAMELSPIN. Although the maximum ROE effect in this experiment still depends on the product of $\omega$ and $\tau_{\mathrm{c}}$, the ROE effect is always positive, and consequently negative cross-peaks are obtaincd. The cross-peak intensity increases with $\omega \tau_{c}$, which means that large, slowly tumbling molecules yield the most intense cross-peaks. The absence of a zero point in the intensity versus $\omega \tau_{c}$ curve makes ROESY suitable for analyses of intermediate sized molecules, like oligosaccharides. ROESY also provides a means to discriminate between real ROE cross-peaks and crosspeaks created by spin diffusion (positive cross-peak), although this kind of magnetization relay is not prominent in ROESY. Nevertheless ROESY is not often used for quantitative ${ }^{1} \mathrm{H} \cdot{ }^{1} \mathrm{H}$ distance determinations, because ROESY cross-peaks 
are influenced by several non-relaxation effects, such as the offset dependence and the HOHAHA type magnetization transfer.

ROESY peak intensities decrease as a function of the resonance frequencies relative to the carrier frequency of the spin-lock pulse. The theoretical basis of this effect, which is referred to as the offset dependence, is fully understood. Therefore, the offset dependence of ROESY spectra is not necessarily an obstacle for quantitative analysis.

Since ROESY is a spin-lock technique, like HOHAHA spectroscopy, magnetization can be transferred through scalar couplings, which gives rise to positive cross-peaks. This effect can introduce two types of error in the analysis: $(a)$ in the case when the negative ROESY cross-peak intensity of two protons is decreased by the addition of the positive HOHAHA contribution, the calculated distance will be overestimated; (b) in the case when two spins have a strong ROE interaction and one of them has also a fairly good Hartmann-Hahn match with another proton, a spurious ROE is obtained. This ROE gives rise to an underestimate of the distance. In order to cope with these problems, the CROSREL program ${ }^{7}$ provides a multi-spin implementation of the two-spin estimation of the HOHAHA effect described by $\mathrm{Bax}^{8}$. Since this approximation is only valid for small HOHAHA transfers, the experimental conditions should be chosen in order to minimize these effects ${ }^{7}$. Therefore, the HOHAHA effect also need not be a prohibitive factor for quantitative ROESY analysis.

Multi-spin and non-relaxation effects in ROESY spectra are accounted for in the full relaxation matrix calculations performed by $\mathrm{CROSREL}^{7}$, allowing quantitative analysis.

From the spectra, diagonal and cross-peak intensities can be measured. Interproton distances can be obtained from cross-peak intensities by using known distances for scaling, either via the initial rate approach or a full matrix method ${ }^{9}$. From these distances, the spatial structure of the molecule can be derived, provided that enough distances can be obtained to establish the values of most, important conformational degrees of freedom. For saccharides, these will typically be the $\phi$ and $\psi$ torsional angles in the glycosidic linkage. Two main problems can occur. Firstly, only a single short interproton distance (sometimes two) may be obtained, which is not sufficient to determine $\phi$ and $\psi$ uniquely. Secondly, the molecule may not have fixed $(\phi, \psi)$ angles and may rapidly fluctuate around average $(\phi, \psi)$ angles, or else several conformations with completely different average $(\phi, \psi)$ angles may simultaneously be present in solution. As the procedures described above will yield average interproton distances, it cannot be decided whether the observed distances correspond to one single conformation or whether the observed distances are the result of an average of two or more conformations.

The approach to incorporate the experimental interproton distances as NOEconstraints in an energy minimisation or MD simulation ${ }^{9}$ does not seem appropriate for saccharides due to the multi-conformation problem. It could yield an 
average conformation that never actually exists. Therefore, independent MD simulations are performed from which ROESY specta are calculated. Several $(\phi, \psi)$ conformations can be combined in order to obtain acceptable agreement with the experimental spectra.

\section{EXPERIMENTAL}

Materials.-Methyl $\beta$-cellobioside was obtained commercially and $\left({ }^{2} \mathrm{H}_{3}\right)$ methyl $\beta$-cellobioside was synthesized from cellobiose ${ }^{10}$, with $\mathrm{Hg}(\mathrm{CN})_{2}$ as a promoter. The $\left({ }^{2} \mathrm{H}_{3}\right)$ methyl compound was used in order to prevent a high $t_{1}$-noise ridge in $2 \mathrm{D}$ NMR experiments at the methyl frequency in the middle of the bulk region.

NMR spectroscopy. $-{ }^{1} \mathrm{H}$ and ${ }^{13} \mathrm{C}$ NMR spectra of methyl $\beta$-cellobioside solutions in $\mathrm{D}_{2} \mathrm{O}$ were recorded at various temperatures. The $\left({ }^{2} \mathrm{H}_{3}\right)$ methyl compound was used in all 2D ${ }^{1} \mathrm{H}$ NMR experiments. Prior to NMR spectroscopy, the sample was exchanged in $\mathrm{D}_{2} \mathrm{O}(99.8 \% \mathrm{D})$ and then lyophilized, finally using $450 \mu \mathrm{L}$ of high quality $\mathrm{D}_{2} \mathrm{O}(99.96 \% \mathrm{D})$. The sample concentration was ca. $10 \mathrm{mM}$ for the ${ }^{1} \mathrm{H}$ experiments and ca. $200 \mathrm{mM}$ for the ${ }^{13} \mathrm{C}$ experiments.

${ }^{1} \mathrm{H}$ NMR spectra were recorded with a Bruker AM-600 (SON hf-NMR facility) and a Bruker AM-500 (Department of NMR Spectroscopy, Utrecht University) at 600 or $500 \mathrm{MHz}$, respectively. ${ }^{13} \mathrm{C}$ NMR spectra were recorded with a Bruker AC-300 (Department of Organic Chemistry, Utrecht University) at $75 \mathrm{MHz}$ at $27^{\circ} \mathrm{C}$. The processing of the NMR data was done on a local $\mu \mathrm{VAX} / \mathrm{VMS}$ cluster using the TRITON 2D-3D NMR program (R. Kaptcin and R. Boclens, Department of NMR Spectroscopy, Utrecht University).

Proton chemical shifts are expressed in ppm downfield from internal sodium 4,4-dimethyl-4-silapentane-1-sulphonate (DSS), but were actually measured by reference to internal acetone $(2.225 \mathrm{ppm})$. Carbon chemical shifts are expressed in ppm downfield from internal acetone $(31.55 \mathrm{ppm})$.

${ }^{13} \mathrm{C}$ NMR $T_{1}$-relaxation values were determined by the aperiodic saturation recovery method (standard Bruker program ASATREC.AU) with a fit for the recovered intensity. Spectra were recorded with a 1.0-s aperiodic saturation pulse and 15 evenly distributed recovery times ranging from 0.102 to $0.802 \mathrm{~s}$ preceding a $90^{\circ}$ observation pulse. In addition, a fully relaxed ${ }^{13} \mathrm{C}$ NMR spectrum was recorded with a repetition time of $2.82 \mathrm{~s}$ in order to determine the initial magnetization. The interpretation of the $T_{1}$ values in terms of rotational mobility was according to ref 11. Briefly, for carbohydrates in solution, the ${ }^{13} \mathrm{C}$ relaxation is governed by dipolar coupling between covalently bonded ${ }^{1} \mathrm{H}$ and ${ }^{13} \mathrm{C}$ spins. Assuming isotropic rotational motion, the $T_{1}$-relaxation time is described by:

$$
\begin{aligned}
\frac{1}{T_{1}^{\mathrm{c}}}= & \left(\frac{\mu_{0}}{4 \pi}\right)^{2} \cdot \frac{\hbar^{2} N \gamma_{\mathrm{C}}^{2} \gamma_{\mathrm{H}}^{2}}{10 r_{\mathrm{C}-\mathrm{H}}^{6}} \\
& \cdot\left\{\frac{\tau_{\mathrm{c}}}{1+\left(\omega_{\mathrm{H}}-\omega_{\mathrm{C}}\right)^{2} \tau_{\mathrm{c}}^{2}}+\frac{3 \tau_{\mathrm{c}}}{1+\omega_{\mathrm{C}}^{2} \tau_{\mathrm{c}}^{2}}+\frac{6 \tau_{\mathrm{c}}}{1+\left(\omega_{\mathrm{H}}+\omega_{\mathrm{C}}\right)^{2} \tau_{\mathrm{c}}^{2}}\right\}
\end{aligned}
$$


where $\mu_{0}$ is the magnetic permeability of vacuum, $\hbar$ is Planck's constant divided by $2 \pi, \gamma_{\mathrm{C}}$ and $\gamma_{\mathrm{H}}$ are the ${ }^{13} \mathrm{C}$ and ${ }^{1} \mathrm{H}$ gyromagnetic ratio, respectively, $\tau_{\mathrm{c}}$ is the rotational correlation time, $r_{\mathrm{C}-\mathrm{H}}$ is the proton-carbon distance, and $N$ is the number of covalently attached protons. In this analysis, every $r_{\mathrm{C}-\mathrm{H}}$ was assumed to be $1.1 \AA$. Since eq 1 cannot be solved analytically for $\tau_{c}$, a numerical approach has been used.

${ }^{1} \mathrm{H}-{ }^{1} \mathrm{H}$ 2D ROESY spectra were measured at $10^{\circ} \mathrm{C}$ with mixing times of 50 , $100,150,200,300$, or $400 \mathrm{~ms}$ at $600 \mathrm{MHz}$. Low temperatures were used in order to increase the rotational correlation time $\tau_{c}$ and thus to increase the ROESY intensity. The sample was not spun. The carrier frequency was placed far downfield of the spectrum at $5.75 \mathrm{ppm}$; the spin lock period involved a simple continuous $r f$ pulse $\mathrm{e}^{6,12}$ with a field strength of $2500 \mathrm{~Hz}$ corresponding to a $90^{\circ}$ pulse length of $100 \mu \mathrm{s}$. The spectral width was $2994 \mathrm{~Hz}$ in both dimensions. Spectra were measured in the phase sensitive mode, using TPPI ${ }^{13-15}$ in order to create amplitude modulation in $t_{1}$. The $t_{1}$ period was incremented from $83.5 \mu \mathrm{s}$ to 85.5 ms. For each ROESY experiment, 512 FIDs of 2048 data points, 16 scans each, were collected. The six data matrices were zero-filled and multiplied in both dimensions with a $\pi / 2$ phase-shifted sine function prior to Fourier transformation (FT). After the first FT of the data in the $\omega_{2}$ domain, a third-order polynomial baseline correction was applied on the data sets, fitted on selected empty regions of the spectrum in order to minimize $t_{1}$ noise. Only the informative high-field halves of the spectra, which contain all the information, were stored and processed in the $\omega_{1}$ domain. The same procedure was used after the second FT and therefore only the essential high-field quadrants of the data sets were stored, obtaining $1024 \times 1024$ real data matrices. A third-order polynomial baseline correction was applied to $2 \mathrm{D}$ spectra in both dimensions to ensure a flat baseplane for unbiased peak integration.

Peak integration was performed by summation of the intensity in a defined area around the center of the peak. The overlapping or almost overlapping peaks of $\mathrm{H}-3,4,5$ of both residues are integrated as resulting from one proton.

\section{METHODS}

$M D$ simulations.-The MD simulations on methyl $\beta$-cellobioside were performed using the program GROMOS ${ }^{16}$ and its standard force field for carbohydrates ${ }^{17}$. The united atom approach was used for aliphatic carbon atoms. Positions of the corresponding hydrogen atoms were calculated after simulation, by using ideal tetrahedral geometries and a $\mathrm{C}-\mathrm{H}$ bond length of $1.1 \AA$. The methyl $\beta$-cellobioside molecule was placed in a periodic computational box containing 358 water molecules. The simulations were performed ${ }^{18}$ at constant temperature $\left(27^{\circ} \mathrm{C}\right)$ and pressure $(1 \mathrm{~atm})$. Trajectories of the methyl $\beta$-cellobioside molecule were stored every $0.02 \mathrm{ps}$. The calculations were performed on a local $\mu \mathrm{VAX} / \mathrm{VMS}$ cluster and on a Convex 120. 
Initial rate analysis. - In order to translate ROESY peak intensities into interproton distances, the initial rate approach was used, for which implicitly isotropic rotational motion is assumed. Prior to this analysis, the H-6pro-S-H-6pro-R and H-6' pro- $S-H-6$ ' pro- $R$ cross-peaks of methyl $\beta$-cellobioside were modified to compensate for an estimated 4\% HOHAHA transfer at each mixing time ${ }^{7}$. All cross-peak intensities were corrected for the offset dependence, with a $\sin ^{2}\left(\theta_{i}\right) \times$ $\sin ^{2}\left(\theta_{\mathrm{j}}\right)$ factor, which is an allowed correction, since the NOESY contribution to the cross-relaxation is negligible for this molecule at $500 \mathrm{MHz}^{7}$. The diagonal peaks were corrected with $\sin ^{2}\left(\theta_{i}\right)$, which is the approximate offset dependence in case of initial build-up rates ${ }^{7}$. The build-up curves were fitted to a quadratic function, and initial build-up rates were determined as the linear coefficients of these functions. Interproton distances were calculated under the assumption of isotropic tumbling by relating their initial build-up rate to that of proton pairs at known distances by the equation:

$r_{\mathrm{ij}}=r_{0}\left(\frac{a_{0}}{a_{\mathrm{ij}}}\right)^{1 / 6}$

where $r_{\mathrm{ij}}$ is the distance to be determined, $r_{0}$ is the known distance, $a_{\mathrm{ij}}$ is the initial rate of a pair of protons at an unknown distance, and $a_{0}$ is the initial rate of a pair of protons at a known distance ${ }^{19,20}$. Intraresidue proton pairs served as references for the distance determination.

CROSREL analysis. - Full relaxation matrix calculations were performed by the CROSREL program ${ }^{7}$, with incorporation of the HOHAHA effect in all theoretical spectra. The noise level was estimated at $0.5 \%$ of the initial magnetization. Several input model distance matrices were derived from MD simulations. The $\mathbf{M}_{0}$-scaling method has been used in all cases presented in this paper. Therefore, the experimental data were normalized to the initial magnetization at $\tau_{\mathrm{m}}=0$, by dividing each peak in a column by the estimated $\mathbf{M}_{0}(\mathbf{i})$ associated with that column. $\mathbf{M}_{0}(i)$ of proton $i$ is estimated by extrapolation of the integrated column intensity ( $\omega_{2}$ constant) to $\tau_{\mathrm{m}}=0$. The calculated spectrum is similarly normalized within the CROSREL program.

The spectrum is calculated from:

$\mathbf{A}\left(\tau_{\mathrm{m}}\right)=\exp \left[-\mathbf{R} \tau_{\mathrm{m}}\right]$

The relaxation matrix $\mathbf{R}$ contains all interproton distances $r_{\mathrm{ij}}$, the rotation correlation time $\tau_{c}$, and the spectrometer frequency $\omega$ through the spectral densities and the leakage relaxation rate, $R_{L}$, which accounts for non-dipole-dipole relaxation. The general expression for the spectral density function, which includes parameters to describe anisotropic and internal motions, as used in CROSREL, is:

$J_{\mathrm{n}}(\omega)=r_{\mathrm{ij}}^{-6} \cdot \frac{\tau_{\mathrm{c}}}{1+n^{2} \omega^{2} \tau_{\mathrm{c}}^{2}} \cdot S^{2}$

where $S^{2}$ is the optional generalized order parameter ${ }^{21}$. The values for $r_{i j}, \tau_{c}$, and $S^{2}$ can be different for each ${ }^{1} \mathrm{H}-{ }^{1} \mathrm{H}$ vector. Here $S^{2}$ is set to 1 , because its effect is 
completely included in ${ }^{1} \mathrm{H}-{ }^{1} \mathrm{H}$ specific $\tau_{\mathrm{c}}$ values (see below). For a rigid isotropically rotating molecule, the rotation correlation time $\tau_{\mathrm{c}}$ can be obtained from calculation of the correlation function, as obtained from MD simulations ${ }^{7}$ :

$$
\begin{aligned}
C(t) & =\left\langle\frac{1}{2}\left(3 \cos ^{2}(\Delta \theta)-1\right)\right\rangle_{0} \\
& =\exp \left(-t / \tau_{\mathrm{c}}\right)
\end{aligned}
$$

where $\Delta \theta$ is the angle between the directions of a vector in the molecule at times 0 and $t$.

When the molecule is not rigid and rotating anisotropically, then, for each ${ }^{1} \mathrm{H}-{ }^{1} \mathrm{H}$ vector, the correlation function has to be calculated:

$C^{\mathrm{HH}}(t)=\left\langle\frac{1}{2}\left(3 \cos ^{2}\left(\Delta \theta^{\mathrm{HH}}\right)-1\right)\right\rangle_{0}$

This $C^{\mathrm{HH}}(t)$ is the product of an internal and overall correlation function, with correlation times $\tau_{\mathrm{i}}$ and $\tau_{0}$, respectively.

$C^{\mathrm{HH}}(t)=C_{\mathrm{I}}(t) \cdot C_{0}(t)=\left[\left(1-S^{2}\right) \exp \left(-t / \tau_{\mathrm{i}}\right)+S^{2}\right] \cdot \exp \left(-t / \tau_{0}\right)$

Here the $C^{\mathrm{HH}}(t)$, due to the specific time scales of internal and overall motions, will be approximated:

$C^{\mathrm{HH}}(t) \approx \exp \left(-t / \tau_{\mathrm{c}}^{\mathrm{HH}}\right)$

When these individual $\tau_{c}$ values are used in CROSREL, it will be referred to as the anistropic model, whereas the use of a uniform $\tau_{c}$ will be referred to as the isotropic model. The goodness of fit of the theoretical and experimental peak intensities is expressed in a weighted residual factor, $R_{\mathrm{w}}$, similar to $\mathrm{X}$-ray crystallography ${ }^{7}$. In the following sections, two strategies for distance averaging will be used. Averaging the distance as $r=\left[\left\langle r^{-3}\right\rangle\right]^{-1 / 3}$ is being used for fast conformational changes, such as the rotation of methyl groups. The consecutive conformations of MD simulations are also averaged in this way. Averaging the distance as $r=\left[\left\langle r^{-6}\right\rangle\right]^{-1 / 6}$ is being used for slow conformational changes.

\section{RESULTS AND DISCUSSION}

In order to assess the conformational characteristics of methyl $\beta$-cellobioside in aqueous solution, MD simulations have been carried out, and a build-up series of ROESY NMR spectra has been acquired. The results have been combined and analysed with the initial rate method and with the CROSREL program ${ }^{7}$. The main focus is on the glycosidic torsional angles $(\phi, \psi)$, and the conformations of the hydroxymethyl groups have also been evaluated.

In the literature, ample information on the $(\phi, \psi)$ energy surface of the methyl $\beta$-cellobioside molecule is available from $M M$ calculations ${ }^{5,22-25}$. The results are all located in four minimal energy regions of the $(\phi, \psi)$ surface. In most of these studies, several minimum energy conformations have been calculated. The $(\phi, \psi)$ 

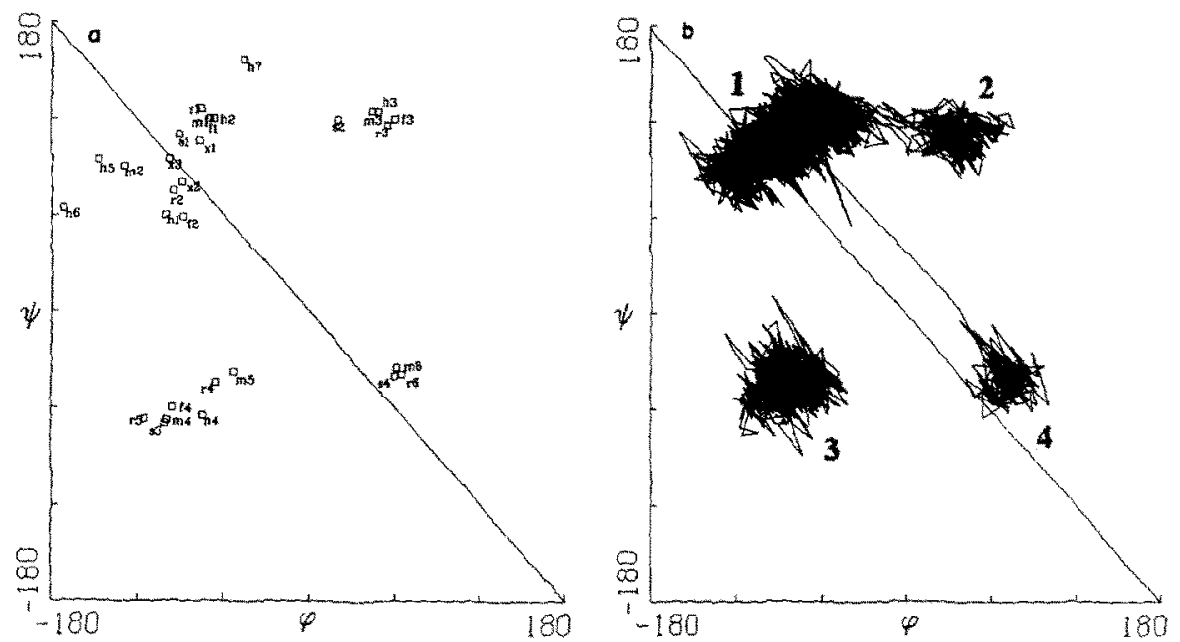

Fig. 2. Conformation space of $(\phi, \psi)$ in degrees of the glycosidic linkage of methyl $\beta$-cellobioside. (a) Minimal energy conformations from $M M$ calculation and data from crystal structures ( $\mathrm{x}$ ). Marks $\mathrm{f}, \mathrm{h}, \mathrm{m}$, 5, and $\mathrm{r}$ indicate minima from refs $22,24,25,5$, and 23 , respectively. (b) Trajectories of five MD runs.

space was searched, either by keeping all remaining internal coordinates fixed, or by calculating a fully relaxed potential energy surface ${ }^{22}$. However, even in the latter case, only a limited number of exocyclic torsional angles (hydroxyl and hydroxymethyl groups) were considered. A more rigorous approach has been applied recently ${ }^{23}$. A prudent-ascent algorithm ensures that those conformations of the exocyclic groups are found that yield the lowest energy at each $(\phi, \psi)$. The $(\phi, \psi)$ values of the minimum energy conformations from the literature are displayed in Fig. 2a. Some authors find a number of minima in the same region, caused partly by different choices of exocyclic torsional angles. The crystal structures of cellobiose ${ }^{26}$ and methyl $\beta$-cellobioside ${ }^{27}$, which are marked in Fig. 2a $x 1$ and $x 2$, respectively, and of the two celluloses ${ }^{1,3}$, marked $x 3$, are found in region 1 . Conformations in this region correspond to the extended conformation of cellulose, and lie near a 2-fold screw axis that can be drawn through the molecule when it has equal $\phi$ and $\psi$ values. Additionally, regions 2, 3, and 4 are characterized by different torsional angles $(\phi, \psi)$, as is indicated in Fig. 2a, and correspond to folded conformations. The data from the literature can be subdivided roughly into two categories. The first category comprises those in which hydrogen bond interactions are properly accounted for by the MM force field ${ }^{5,23,24}$. They all find the minimum in region 2 to have the lowest energy. The other category involves force fields, which are derived without special attention to a good description of bydrogen bonds ${ }^{22,25}$, yielding the lowest energy conformation in region 1 . The reason for this difference is that more hydrogen bonds can be created in region 2 . Because hydrogen bonds lower the energy considerably, force fields describing these interactions properly will find them. It is probable that in a solvent like 
water, these intramolecular hydrogen bonds will not survive ${ }^{18,30}$, and that thereby the importance of region 2 will be reduced.

For the rotamer distribution of the hydroxymethyl group, NMR data are available. It is known from measurements of ${ }^{3} J_{\mathrm{HH}}$ coupling constants that these distributions are $g g: g t: t g=0.60: 0.40: 0.00$ for the reducing-end glucose residue, and $g g: g t: t g=0.49: 0.50: 0.01$ for the terminal residue, respectively ${ }^{28}$. In order to enter accurate models into the CROSREL program ${ }^{7}$, the rotamer distribution should be correct.

MD simulations: conformations and interproton distances.--Here, MD simulations in water have been carried out, which were started in each of the four conformational regions. The trajectories of the simulations (steps of $0.2 \mathrm{ps)} \mathrm{in}$ these four regions of the $(\phi, \psi)$ surface are displayed in Fig. 2b. The simulation started in region 1 was continued for $500 \mathrm{ps.} \mathrm{Once,} \mathrm{for} \mathrm{a} \mathrm{short} \mathrm{period} \mathrm{of} \mathrm{time} \mathrm{of} \mathrm{ca.}$ $20 \mathrm{ps}$, the molecule visited region 2 . A separate simulation started in region 2 resulted in a transition to 1 after ca. 40 ps, suggesting that region 2 does not comprise stable conformations. Two simulations were started in region 3 of 50 and 150 ps, respectively. Here, the average conformation seems to be stable (see Fig. 2b). A run in region 4 resulted in a transition after $40 \mathrm{ps,}$, again to region 1 . Analysis of time-averaged energies, although hampered by considerable noise, clearly rejects conformations in region 4 , because the total energy of the system is higher than that of the average conformation in region 1 by more than $3 \sigma$ (50 $\mathrm{kJ} /$ mole). The energy differences resulting from simulations in regions 1,2 , and 3 are of the order of $\sigma$. As the average conformation in region 4 , and probably also 2 , is unstable, it is especially that in region 3 which is of interest for a possible solution structure.

It is striking that the spread in $(\phi, \psi)$ in each region, resulting from the data in Fig. 2a, is comparable to spread obtained from the MD simulations, as displayed in Fig. 2b. Region 1 shows considerable flexibility. From MD simulations of cellobiose in vacuum at $127^{\circ} \mathrm{C}$, this flexibility was already suggested ${ }^{29}$. Region 1 consists of two minima, rather than one minimum, like all $\mathrm{MM}$ calculations and the crystal structures of $\beta$-cellobiose and methyl $\beta$-cellobioside indicate, with hardly any barrier in between. Conformational freedom in region 3 is much more restricted and this region appears to be separated from 1 by a large barrier, since no transitions occur. Averaged interresidue ${ }^{1} \mathrm{H} \cdots{ }^{1} \mathrm{H}$ distances $\left(r=\left[\left\langle r^{-3}\right\rangle\right]^{-1 / 3}\right)$ obtained from the simulations in regions 1 and 3 are quite different, so that ROESY NMR spectra can give a decisive answer as to whether conformations in region 3 exist in solution.

In addition, the conformations of the hydroxymethyl groups need discussion. The $\omega$ and $\omega^{\prime}$ trajectories during the 500-ps simulation in region 1 are shown in Fig. 3. Several transitions of both hydroxymethyl groups occurred. However, the number of transitions is not large enough to ensure a proper distribution of the three staggered hydroxymethyl group conformations, in particular the $\omega$ distribution for which $g g: g t: t g=0.78: 0.13: 0.09$ is found. This group is "trapped" in the 

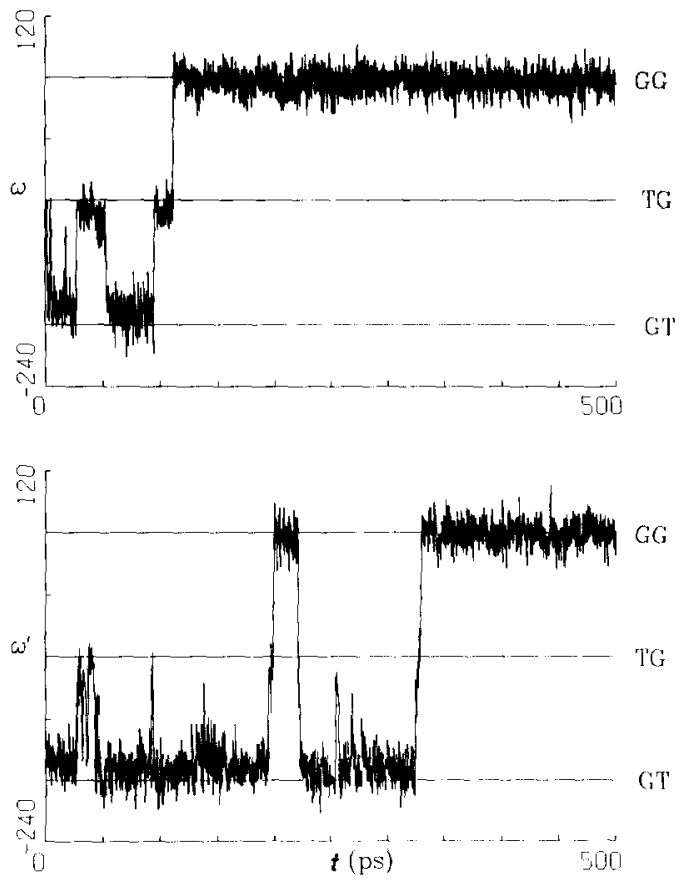

Fig. 3. MD trajectories of the torsional angles $\omega$ and $\omega^{\prime}$ in degrees of methyl $\beta$-cellobioside during 500 ps.

GG conformation for ca. 390 ps. It has already been discussed before ${ }^{30}$ that the barrier to leave or reach the GG conformation is larger than that between the GT and TG conformations. Therefore, the above result does not necessarily reflect a very low energy conformation. The $\omega^{\prime}$ torsional angle shows more transitions leading to a distribution of $g g: g t: t g=0.38: 0.56: 0.06$. These results deviate from the experimental values. For this reason, the experimental distributions for the hydroxymethyl group conformations have been used to create possible interproton distance matrices. Four different matrices have been used in the CROSREL calculations. $\mathbf{D}_{\mathrm{A}}$ : the interproton distances were derived from two parts of the trajectory in region $\mathbf{1}$, one in which both hydroxymethyl groups were in the GG conformation ( $100 \mathrm{ps}$ of data) and the other with both hydroxymethyl groups in the GT conformation ( $35 \mathrm{ps}$ of data). In view of the experimental value of $t g$, the TG conformation was not included in this distance matrix. Within these two sets, the distances were averaged as $r=\left[\left\langle r^{-3}\right\rangle\right]^{-1 / 3}$, whereas the two sets were combined using $r=\left[\left\langle r^{-6}\right\rangle\right]^{-1 / 6}$ with a population of $g g: g t=0.55: 0.45$, being the average of the experimental rotamer distributions. $\mathbf{D}_{\mathrm{B}}$ : a similar procedure was applied to the trajectory in region 3 , using 50 ps of data of a $2 *$ GG conformation and 20 ps of a $2 *$ GT conformation. Strictly, one should use all four combinations of $\omega$ and $\omega^{\prime}$, but in fact no short distances would be affected by taking the GT/GG and GG/GT conformations into account. $\mathbf{D}_{\mathrm{C}}$ : the whole trajectory of $500 \mathrm{ps}$ in region 1 


\section{TABLE I}

$\tau_{c}$ values (ps) ${ }^{a}$ for the principal axes of inertia and for ${ }^{1} \mathrm{H}-{ }^{1} \mathrm{H}$ vectors of methyl $\beta$-cellobioside as determined from MD data

Principal axes:

\begin{tabular}{llll}
$X$ & $Y$ & $Z^{b}$ \\
\hline 39.7 & 37.9 & 65.0
\end{tabular}

${ }^{1} \mathrm{H}-{ }^{1} \mathrm{H}$ vectors:

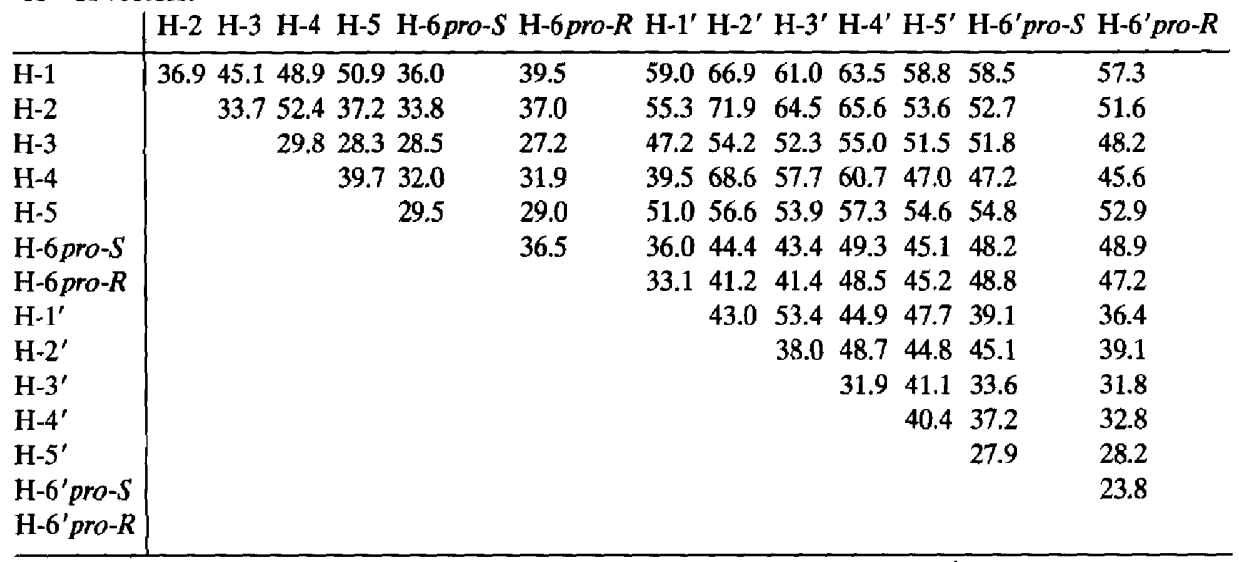

The $\tau_{c}$ values are obtained from a least-squares fit to $C(t)=\exp \left(-t / t_{c}\right)^{b}$ The axes are ordered according to the moments of inertia such that $X$ has the largest and $Z$ has the smallest moment.

has been used $\left(r=\left[\left\langle r^{-3}\right\rangle\right]^{-1 / 3}\right)$ and to arrive at better statistics, especially for the hydroxymethyl group conformations, equivalent distances within both residues were averaged by $r=\left[\left\langle r^{-6}\right\rangle\right]^{-1 / 6}$, arriving at a distribution of $g g: g t: \operatorname{tg}=$ $0.58: 0.35: 0.08$. This is allowed because experiment shows that both hydroxymethyl groups have a similar distribution. $\mathbf{D}_{\mathrm{D}}$ : again the whole region 1 trajectory has been used but now, to arrive at a proper hydroxymethyl group distribution, the distances involving all $\mathrm{H}-6$ atoms were replaced by those from matrix $\mathbf{D}_{\mathrm{A}}$.

These interproton distance matrices were entered into the CROSREL program, in order to find the most accurate molecular model for methyl $\beta$-cellobioside in solution. Special attention was given to the significance of the average conformation in region 3 , which is regarded as the only candidate to allow back folding of cellulose chains.

MD simulation: analysis of the overall tumbling.-In the analysis of NOESY or ROESY spectra, isotropic tumbling of molecules is usually assumed. However, an analysis of the rotation of methyl $\beta$-cellobioside in terms of the rotational correlation functions of the three principal axes of inertia from the 500-ps run in region 1 suggested anisotropic motion for this molecule ${ }^{7}$. The correlation time $\tau_{\mathrm{c}}$ for the $Z$ axis was found to be more than 1.5 times that for the $X$ and $Y$ axes, as is shown in Table I. Both this anisotropy and internal motions can be accounted for by introducing individual $\tau_{\mathfrak{c}}$ values for each ${ }^{1} \mathrm{H}-{ }^{1} \mathrm{H}$ vector in the spectral densities. 


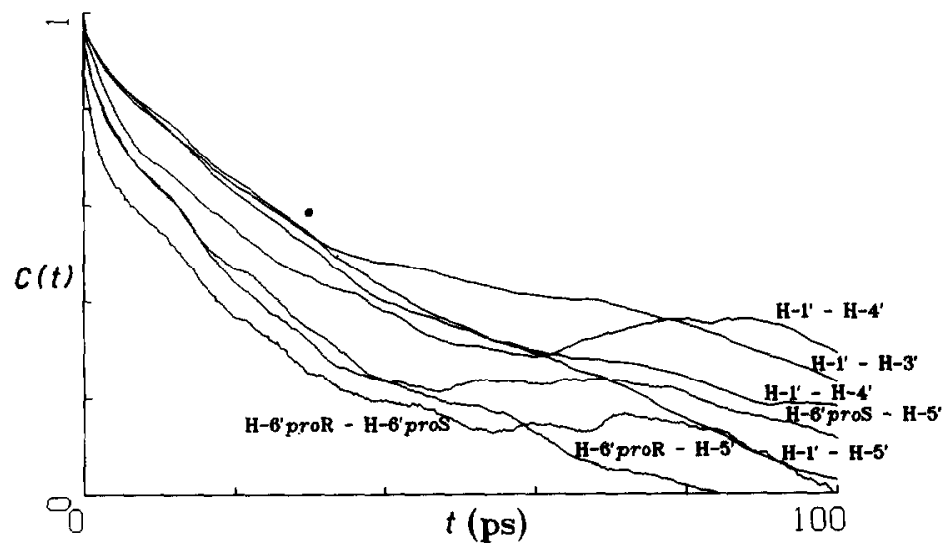

Fig. 4. Correlation functions $C(t)$ of several ${ }^{1} \mathrm{H}-{ }^{1} \mathrm{H}$ vectors of methyl $\beta$-cellobioside obtained from MD simulations.

For all vectors, $C(t)$ from eq 6 has been calculated and fitted to eq 8 , and for some ${ }^{1} \mathrm{H}-{ }^{1} \mathrm{H}$ vectors $C(t)$ up to $100 \mathrm{ps}$ is plotted in Fig. 4. Already, considerable noise is visible so that the fits were carried out using $C(t)$ only up to $50 \mathrm{ps}$. The $\tau_{\mathrm{c}}$ values are listed in Table I. Especially, the vectors involving $\mathrm{H}-6$ atoms have small $\tau_{c}$ values, caused by conformational transitions of the hydroxymethyl groups in addition to rotational flexibility in each staggered conformation. This rotational motion can be analysed in more detail. The orientation of a ${ }^{1} \mathrm{H}-{ }^{1} \mathrm{H}$ vector can be transformed into the molecule-fixed axes system: $\mathbf{v}_{M}=\mathbf{T} \cdot \mathbf{v}_{\mathrm{L}}$, where $\mathbf{T}$ is the matrix composed of the three Euler rotations transforming the laboratory-fixed axes system into the molecule-fixed axes system. When the correlation function is calculated by using eq 6 in the molecule-fixed axes system, the internal correlation function $C_{\mathrm{I}}(t)$ is obtained. Assuming that $C(t)$ is the product of the internal correlation function $C_{\mathrm{I}}(t)$ and the overall correlation function $C_{0}(t)$, according to ref 21 and eq 7 , the latter can be obtained. $C(t)$ with its $\exp \left(-t / \tau_{\mathrm{c}}\right)$ fit, $C_{\mathrm{I}}(t)$, and $C_{0}(t)$ for H-6'pro-R-H-6'pro-S are plotted in Fig. 5. The $C_{0}(t)$ curve corresponds to a $\tau_{\mathrm{c}}$ value of $43 \mathrm{ps}$, which is entirely due to overall anisotropic motion. This is a reaonable value in view of the $\tau_{\mathrm{c}}$ values for the three principal axes. $C_{\mathrm{I}}(t)$ clearly falls to a plateau value $S^{2}$. In fact, the plot suggests the existence of two plateaus, implying two different internal motions. This is in agreement with the idea of a rapid vibrational motion in an $\omega$-potential well and a somewhat slower motion due to conformational transitions. Although a wealth of information on internal motion is available from MD simulations, it has already been stated ${ }^{21}$ that a detailed analysis of this internal motion is not necessary in order to calculate the spectral density function. It is sufficient to represent all internal motions by $C_{1}(t)=S^{2}+(1$ $\left.-S^{2}\right) \exp \left(-t / \tau_{\mathrm{i}}\right) . S^{2}$ and $\tau_{\mathrm{i}}$ can be obtained from a fit to $C_{\mathrm{I}}(t)$. However, even this analysis is not necessary, because $C(t)=\left[S^{2}+\left(1-S^{2}\right) \exp \left(-t / \tau_{\mathrm{i}}\right)\right]$ $\exp \left(-t / \tau_{0}\right)$ can be accommodated in a single $\exp \left(-t / \tau_{\mathrm{c}}\right)$ in the present case, according to approximation 2 in ref 7 (i.e., $\tau_{0} \approx 70$ ps and $\tau_{\mathrm{i}} \approx 10-30 \mathrm{ps}$ ). 


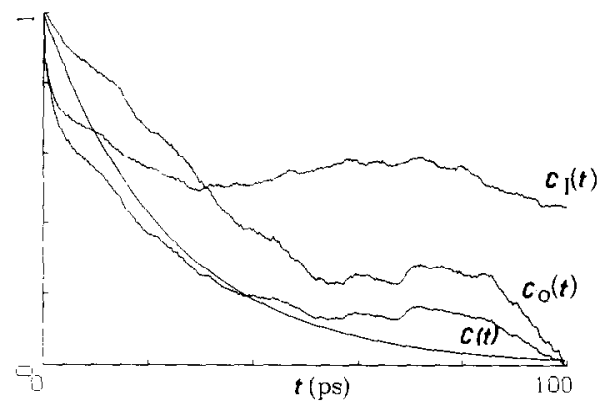

Fig. 5. Correlation functions $C(t), C_{0}(t)$, and $C_{\mathrm{I}}(t)$ of the H-6'pro-S-H-6'pro-R vector for methyl $\beta$-cellobioside obtained from MD simulations.

This simplification was checked by comparing relative ROEs for two interacting proton spins calculated by using the complete correlation functions according to eq 7 , with those acquired by using $\exp \left(-t / \tau_{\mathrm{c}}\right)$ obtained from a fit to $C(t)$ up to $50 \mathrm{ps}$. This comparison was made for $\tau_{0}=70$ and $\tau_{i}=30$ ps while $S^{2}$ was varied from 0 to 1 . In Fig. 6, the solid line represents the calculated exact ROE; the squares represent the ROEs calculated using the approximation. The worst agreement was obtained for $S^{2}=0.5$. The ROE is ca. $15 \%$ too small. This is not a serious discrepancy because it represents a systematic error in all ${ }^{1} \mathrm{H}-{ }^{1} \mathrm{H} \tau_{\mathrm{c}}$ values, which will be removed by fitting the final $\tau_{c}$ to the observed ROEs in the CROSREL program. The resulting $\tau_{\mathrm{c}}$ values will be used as relative $\tau_{\mathrm{c}}$ factors in the CROSREL analysis in the anisotropic model.

By means of the CROSREL program, the significance of the suggested anistropic and internal motions will be evaluated.

NMR spectrascopy: assignment and peak integration.-The complete assignment of the ${ }^{1} \mathrm{H}$ NMR spectrum of methyl $\beta$-cellobioside, as given in Table II, was made by use of 2D HOHAHA and 2D DQF-COSY spectra and is in accordance with earlier findings ${ }^{18}$. In spite of the high spectrometer frequency of $6000 \mathrm{MHz}$, it is

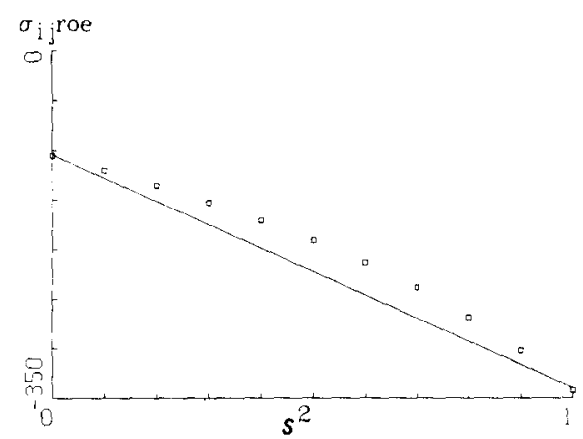

Fig. 6. Comparison of two-spin ROE intensities in arbitrary units calculated by the exact correlation function $C(t)$ (solid line) and by the single exponential approximation (squares). 
TABLE II

${ }^{1} \mathrm{H}$ and ${ }^{13} \mathrm{C}$ assignments of methyl $\beta$-cellobioside in $\mathrm{D}_{2} \mathrm{O}$ at $27^{\circ} \mathrm{C}$ : chemical shifts are expressed relative to internal acetone at 2.225 and $31.55 \mathrm{ppm}$, respectively

\begin{tabular}{|c|c|c|c|c|c|c|c|c|}
\hline${ }^{1} \mathrm{H}$ Assignment & 1 & 2 & 3 & 4 & 5 & 6 pro-S & 6 pro-R & $\mathrm{OMe}$ \\
\hline $\begin{array}{l}\text { Reducing-end } \\
\text { residue } \\
\text { Terminal residue }\end{array}$ & $\begin{array}{l}4.506 \\
4.401\end{array}$ & $\begin{array}{l}3.303 \\
3.312\end{array}$ & $\begin{array}{l}3.630 \\
3.506\end{array}$ & $\begin{array}{l}3.635 \\
3.413\end{array}$ & $\begin{array}{l}3.595 \\
3.485\end{array}$ & $\begin{array}{l}3.991 \\
3.913\end{array}$ & $\begin{array}{l}3.817 \\
3.731\end{array}$ & 3.575 \\
\hline${ }^{13} \mathrm{C}$ Assignment & 1 & 2 & 3 & 4 & 5 & 6 & & $\mathrm{OMe}$ \\
\hline $\begin{array}{l}\text { Reducing-end } \\
\text { residue } \\
\text { Terminal residue }\end{array}$ & $\begin{array}{l}104.4 \\
103.9\end{array}$ & $\begin{array}{l}74.5 \\
74.2\end{array}$ & $\begin{array}{l}75.6 \\
76.8\end{array}$ & $\begin{array}{l}80.0 \\
70.8\end{array}$ & $\begin{array}{l}76.1 \\
77.3\end{array}$ & $\begin{array}{l}61.4 \\
61.9\end{array}$ & & 58.5 \\
\hline
\end{tabular}

not possible to separate all resonances and some serious overlap occurs. The signals of $\mathrm{H}-3,4,5$ of the reducing-end residue of the molecule almost coincide in the region between 3.66 and $3.57 \mathrm{ppm}$ and show strong coupling features. Comparable effects are found in the terminal residue, where $\mathrm{H}-3^{\prime}, 4^{\prime}, 5^{\prime}$ resonate in the small frequency range of 3.53 to $3.40 \mathrm{ppm}$. Although here the overlap is less than in the reducing-end residue, a very strong HOHAHA transfer between these signals must still be expected. For both sets of signals, it is therefore impossible to measure individual cross-peak intensities, and as a consequence they are treated as one single, broad resonance. As an example, the ROESY spectrum of methyl

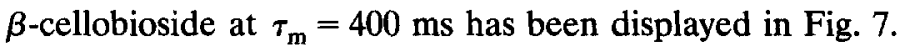

NMR spectroscopy: initial rate analysis. - The initial rate approach ${ }^{19,20}$ provides an easy method to convert cross-peak intensities into interproton distances by estimating the rate of cross-peak intensity build-up at very short mixing times by a fit procedure. The peak intensities of the H-6pro-S to H-6pro- $R$ and the H-6'pro-S to H-6'pro- $R$ peaks and vice versa at the shortest mixing time $\left(\tau_{\mathrm{m}}=0.05 \mathrm{~s}\right)$ were omitted during the fitting procedure because the HOHAHA oscillation had, apparently, not yet damped out.

Interproton reference distances were taken from distance matrix $\mathbf{D}_{\mathrm{C}}$, obtained from the MD simulation of methyl $\beta$-cellobioside in water. Suitable reference distances in a glucose residue are the $\mathrm{H}-1 \cdots \mathrm{H}-3$ and the $\mathrm{H}-1 \cdots \mathrm{H}-5$ distances, respectively. Since the H-3,4,5 signals overlap, a short virtual reference distance can be calculated by eq 9 , which accounts for the observed high intensity cross-peak:

$r=\left\{\sum_{i=1}^{n} r_{i}^{-6}\right\}^{-1 / 6}$

where $n$ is the number of distances to be included. Since the $\mathrm{H}-1 \cdots \mathrm{H}-4$ distance is $4.0 \AA$, its effect on the cross-peak will be very small, and is considered to be negligible. Prior to application of eq 9 , the corresponding $\mathrm{H}-1 \cdots \mathrm{H}-3$ and $\mathrm{H}-$ $1 \cdots \mathrm{H}-5$ distances in each residue were averaged by $r=\left[\left\langle r^{-6}\right\rangle\right]^{-1 / 6}$. Other 


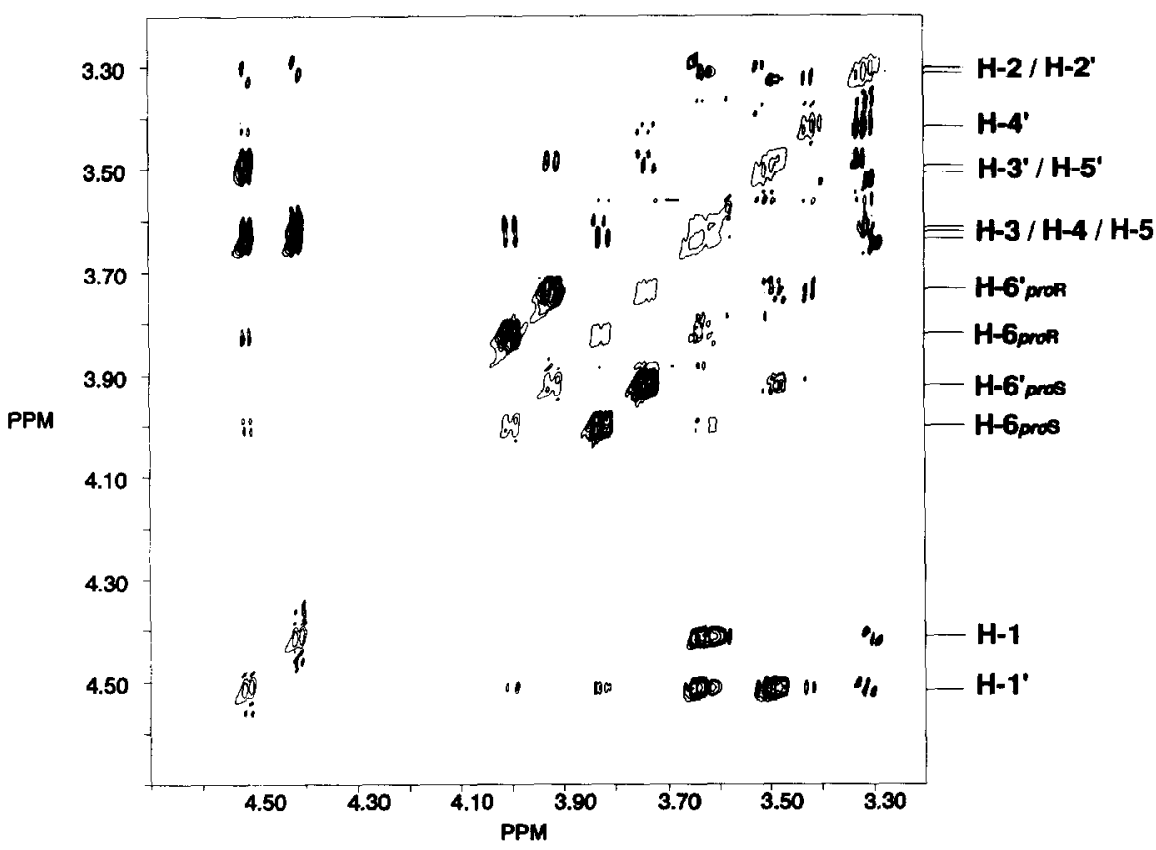

Fig. 7. 2D ROESY spectrum of methyl $\beta$-cellobioside at $10^{\circ} \mathrm{C}$ at $600 \mathrm{MHz}$ and a spin-lock time of 400 ms. The carrier frequency was positioned at $5.75 \mathrm{ppm}$, and the applied field strength was $2500 \mathrm{~Hz}$.

proton pairs that served as references are the HOHAHA-corrected methylene protons in the hydroxymethyl group of each glucose residue.

Every $\mathrm{H}-1-\mathrm{H}-3,5$ and $\mathrm{H}-6$ pro-S $-\mathrm{H}-6$ pro- $R$ build-up rate was used as a reference with the proper distance. In this way, several values were obtained for each interproton distance, to which simple statistics have been applied in order to obtain average distances, including the standard deviation as their estimated errors, which are listed in Table III. The intraresidue distances, which are also used as references, provide excellent checks for the internal consistency. The differences between the values obtained for the different reference distances are ca. $0.1 \AA$, which is considered to be a good agreement. The accuracy of the interglycosidic $\mathrm{H}-1^{\prime} \cdots \mathrm{H}-3,4,5$ distance is regarded as equally good. On the basis of the initial rate data, no conclusion can be drawn with respect to the quality of either reference set. Therefore, the average distances calculated on the basis of both reference sets will be evaluated. This results in an estimated distance $r_{\mathrm{H}-1^{\prime}-\mathrm{H}-3,4,5}=2.16 \AA$. The implications of this value for the glycosidic linkage conformation will be evaluated (see below).

NMR spectroscopy: CROSREL analysis. - The first steps of performing full NOE or ROE relaxation matrix analyses are the determination of the initial molecular model c.q. interproton distances, and the estimation of the leakage relaxation rate $\left(R_{L}\right)$ and the rotational correlation time $\left(\tau_{c}\right)$. Here the molecular model has been obtained from MD simulations. $R_{L}$ cannot be determined experimentally; the 
TABLE III

Build-up rates and distances derived from a ROESY build-up series of methyl $\beta$-cellobioside

\begin{tabular}{|c|c|c|c|c|c|c|}
\hline \multicolumn{2}{|c|}{${ }^{1} \mathrm{H}-{ }^{1} \mathbf{H}$ connectivity } & \multirow{2}{*}{$\begin{array}{l}\text { Initial } \\
\text { rate }\end{array}$} & \multirow{2}{*}{$\begin{array}{l}\text { Reference } \\
\text { distance }(\AA)\end{array}$} & \multirow{2}{*}{$\begin{array}{l}\text { Calculated } \\
\text { distance }(\AA) \\
\text { (ref: } \mathrm{H}-1-\mathrm{H}-3,5)\end{array}$} & \multirow{2}{*}{$\begin{array}{l}\text { Calculated } \\
\text { distance ( } \mathrm{\AA}) \\
\text { (ref: } H-6 \text { pro-S- } \\
\text { H-6pro-R) }\end{array}$} & \multirow{2}{*}{$\begin{array}{l}\text { Calculated } \\
\text { distance }(\AA) \\
\text { (ref: both) }\end{array}$} \\
\hline Intraresidue & & & & & & \\
\hline $\mathrm{H}-1$ & $\mathrm{H}-3,5$ & -0.268 & 2.17 & $2.17 \pm 0.04$ & $2.04 \pm 0.04$ & $2.10 \pm 0.07$ \\
\hline H-3,5 & $\mathrm{H}-1$ & -0.263 & & & & \\
\hline $\mathrm{H}-\mathbf{1}^{\prime}$ & $\mathrm{H}-3^{\prime}, 5^{\prime}$ & -0.229 & & & & \\
\hline $\mathrm{H}-3^{\prime}, 5^{\prime}$ & $\mathrm{H}-\mathbf{1}^{\prime}$ & -0.230 & & & & \\
\hline H-6pro-S & H-6pro-R & -0.513 & 1.80 & $1.92 \pm 0.04$ & $1.80 \pm 0.04$ & $1.86 \pm 0.07$ \\
\hline H-6pro-R & H-6pro-S & -0.460 & & & & \\
\hline$H-6^{\prime}$ pro- $R$ & H-6pro-S & -0.583 & & & & \\
\hline H-6'pro- $R$ & H-6pro-S & -0.542 & & & & \\
\hline \multicolumn{7}{|l|}{ Interresidue } \\
\hline H-1' & H-3,4,5 & -0.197 & & $2.23 \pm 0.04$ & $2.09 \pm 0.04$ & $2.16 \pm 0.07$ \\
\hline $\mathrm{H}-3,4,5$ & $\mathrm{H}-\mathbf{1}^{\prime}$ & -0.227 & & & & \\
\hline
\end{tabular}

correlation time $\tau_{c}$, on the other hand, can be estimated experimentally from ${ }^{13} \mathrm{C}$ NMR $T_{1}$ relaxation measurements or from MD simulations (see previous section). The $T_{1}$ and $\tau_{\mathrm{c}}$ values obtained for methyl $\beta$-cellobioside by ${ }^{13} \mathrm{C}$ NMR analysis are listed in Table IV, including $\left\langle\tau_{\mathrm{c}}\right\rangle$ which can serve as the $\tau_{\mathrm{c}}$ estimate in CROSREL calculations.

Since those $R_{L}$ and $\tau_{c}$ values that give optimal results in NOE/ROE calculations are sought, the CROSREL program ${ }^{7}$ allows optimization of $R_{L}$ and $\tau_{c}$ by means of a grid search. For the distance matrices $D_{A}, D_{C}$, and $D_{D}, R_{L}$ and $\tau_{c}$ have been optimised by using the intraresidue cross-peaks for the isotropic model as well as for the anisotropic model. For the latter, the $\tau_{c}$ values were averaged for corresponding ${ }^{1} \mathrm{H}-{ }^{1} \mathrm{H}$ vectors in each glucose residue, and divided by 65.0 , in order to obtain relative $\tau_{c}$ factors. The $\tau_{c}$ values displayed in Table $I$ are multiplied by these factors in CROSREL to produce the spectral densities. The $\tau_{\mathrm{c}}$

\section{TABLE IV}

${ }^{13} \mathrm{C}$ NMR $T_{1}$ values and corresponding $\tau_{c}$ values determined for methyl $\beta$-cellobioside at $27^{\circ} \mathrm{C}$ and 75 $\mathrm{MHz}^{a}$

\begin{tabular}{|c|c|c|c|c|}
\hline \multirow[t]{2}{*}{ Atom number } & \multicolumn{2}{|c|}{ Reducing-end residue } & \multicolumn{2}{|c|}{ Terminal residue } \\
\hline & $T_{1}(\mathrm{~s})$ & $\overline{\tau_{\mathrm{c}}(\mathrm{ps})}$ & $\overline{T_{1}(\mathrm{~s})}$ & $\overline{\tau_{\mathrm{c}}(\mathrm{ps})}$ \\
\hline 1 & 0.57 & 92.5 & 0.53 & 100.7 \\
\hline 2 & 0.54 & 98.5 & 0.61 & 85.7 \\
\hline 3 & 0.60 & 87.3 & 0.56 & 94.5 \\
\hline 4 & 0.55 & 96.4 & 0.55 & 96.4 \\
\hline 5 & 0.55 & 96.4 & 0.56 & 96.5 \\
\hline 6 & 0.26 & 100.3 & 0.24 & 113.4 \\
\hline
\end{tabular}

$\overline{{ }^{a}\left\langle\tau_{\mathrm{c}}\right\rangle=96 \pm 7 \mathrm{ps}}$ 
values obtained by this procedure range from 100 to $320 \mathrm{ps}$. When isotropic tumbling is assumed, the optimal fit is obtained at $\tau_{c}=150 \mathrm{ps}$. The deviation of the $\tau_{c}$ values obtained directly from the MD data and those obtained by CROSREL fit is not worrisome in view of the numerous approximations made in the MD force field and procedures. However, the relative $\tau_{\mathrm{c}}$ factors will be significantly reliable to be used in CROSREL.

From the narrow range of $\tau_{\mathrm{c}}$ values, derived from ${ }^{13} \mathrm{C}$ NMR $T_{1}$ measurements, it is tempting to conclude that the molecule tumbles isotropically, although $\left\langle\tau_{\mathrm{c}}\right\rangle=96 \mathrm{ps}$ is significantly lower than the CROSREL optimum. This result seems to contradict the rotational correlation times of the three principal axes of inertia, that were determined from MD simulations, which point to anisotropic rotation. Ironically, analysis of the general orientations of ${ }^{1} \mathrm{H}-{ }^{1} \mathrm{H}$ and ${ }^{1} \mathrm{H}-{ }^{13} \mathrm{C}$ vectors reveals that the discrepancy between the two isotropic $\tau_{\mathrm{c}}$ values can be explained to a large extent by anisotropic motion of the molecule. All ${ }^{1} \mathrm{H}-{ }^{13} \mathrm{C}$ vectors are aligned perpendicular to the principal $Z$ axis of inertia, whereas almost all ${ }^{1} \mathrm{H}-{ }^{1} \mathrm{H}$ vectors are aligned along this axis. The only ${ }^{1} \mathrm{H}-{ }^{1} \mathrm{H}$ vectors that are clearly aligned perpendicular to the $Z$ axis are the $\mathrm{H}-3-\mathrm{H}-5$ vectors. The resulting cross-peaks, however, overlap with the H-3-H-4 and $\mathrm{H}-4-\mathrm{H}-5$ cross-peaks, and cannot be analyzed independently. This means that the $\tau_{\mathrm{c}}$ experienced by the ${ }^{1} \mathrm{H}-{ }^{1} \mathrm{H}$ vectors

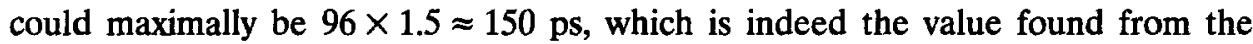
CROSREL fit. An additional factor is the different temperature at which the ROESY $\left(10^{\circ} \mathrm{C}\right)$ and ${ }^{13} \mathrm{C}$ NMR spectra $\left(27^{\circ} \mathrm{C}\right)$ have been acquired, because the mobility of the molecule increases at higher temperatures, which will thereby lower the overall rotational correlation time.

Some typical, observed and calculated ROEs for distance matrices $\mathbf{D}_{\mathrm{A}}$ through $D_{D}$ in both the isotropic and the anisotropic model are plotted in Fig. 8. For matrix $\mathbf{D}_{B}$, the optimal $R_{L}$ and $\tau_{c}$ for $\mathbf{D}_{A}$ have been used. The results of the grid searches are summarized in Table $V$. The difference in the $R_{L}$ value for the different matrices is not regarded as significant in view of the shallow minimum in the $\mathbf{R}_{L}$ domain ${ }^{7}$. For intraresidue distances, the $\mathbf{D}_{C}$ and $\mathbf{D}_{\mathrm{D}}$ matrices appear to be better than $D_{A}$ for methyl $\beta$-cellobioside, as judged from Table $V$. However, the ROEs for intraresidue distances do not show large differences between the various models, as can be seen in Fig. 8. This is reasonable since, with respect to intraresidue distances, these models are more or less the same. Although the effective $\tau_{c}$ values differ considerably, the isotropic and anisotropic models give a similar quality of fit in all matrices.

Since regions 1 and 3 are of interest for possible solution conformations, an attempt has been made to derive the ratio of their average conformations from the ROESY data. Region 1 is characterized by short $\mathrm{H}-\mathbf{1}^{\prime} \cdots \mathrm{H}-4$ distances of $\mathrm{ca}$. 2.0 $\AA$, and relatively long $\mathrm{H}-1^{\prime} \cdots \mathrm{H}-3$ and $\mathrm{H}-1^{\prime} \cdots \mathrm{H}-5$ distances of 4.1 and $3.5 \AA$, respectively. Conformations in region 3 , on the other hand, have two short distances for $\mathrm{H}-1^{\prime} \cdots \mathrm{H}-3$ and $\mathrm{H}-1^{\prime} \cdots \mathrm{H}-5$ of ca. 1.8 and $3.2 \AA$, respectively, and a long $\mathrm{H}-\mathbf{1}^{\prime} \cdots \mathrm{H}-4$ distance of ca. $3.5 \AA$. Since the cross-peaks with $\mathrm{H}-3,4,5$ are 

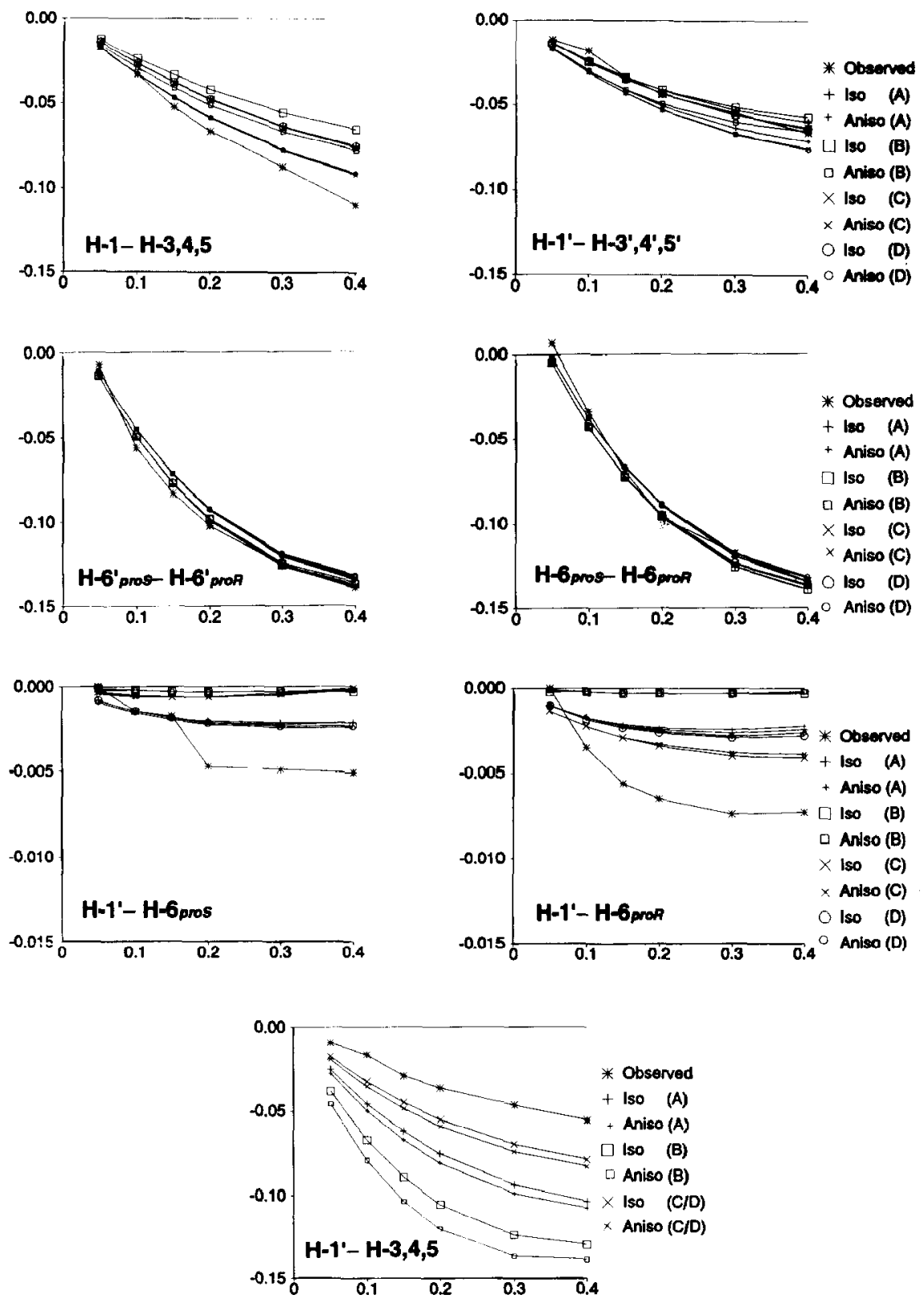

Fig. 8. Build-up curves of ROE intensities relative to $M_{0}$ obtained experimentally and from molecular models by using CROSREL.

treated as originating from one virtual proton, it is not certain that the population of the two average conformations can be determined. The cross-peak intensities for $H-1^{\prime}-\mathbf{H}-3,4,5$ of the various models are plotted in Fig. 8. The observed ROEs are smaller than any of the calculated ROEs. As far as this ROE is concerned, 


\section{TABLE V}

Comparison of several distance matrices by CROSREL $R_{L} / \tau_{c}$ grid search for a ROESY build-up series for $\left({ }^{2} \mathrm{H}_{3}\right)$ methyl cellobioside ${ }^{a}$

\begin{tabular}{|c|c|c|c|c|c|c|}
\hline \multirow[t]{2}{*}{ Dist. matrix } & \multicolumn{3}{|c|}{ Isotropic } & \multicolumn{3}{|c|}{ Anisotropic $\left(\left\langle\tau_{c}\right\rangle\right)$} \\
\hline & $\overline{\mathbf{R}_{\mathrm{L}}}$ & $\tau_{c}$ & $\mathbf{R}_{\mathbf{w}}$ & $\mathbf{R}_{\mathrm{L}}$ & $\tau_{c}$ & $\mathbf{R}_{\mathbf{w}}$ \\
\hline$\overline{\mathbf{D}_{\mathrm{A}}}$ & 0.20 & 150 & 0.0647 & 0.20 & 290 & 0.0650 \\
\hline $\mathbf{D}_{\mathbf{C}}$ & 0.25 & 150 & 0.0629 & 0.25 & 290 & 0.0624 \\
\hline $\mathbf{D}_{\mathrm{D}}$ & 0.25 & 150 & 0.0642 & 0.25 & 290 & 0.0632 \\
\hline
\end{tabular}

${ }^{a} R_{w}$ is calculated from intraresidue cross-peaks only. $R_{L}$ is expressed in $s^{-1}$ and $\tau_{v}$ in ps. $M_{0^{-}}$-scaling and HOHAHA estimate incorporation were used in all cases.

model $\mathbf{D}_{\mathrm{B}}$ is the worst. Two other interglycosidic contacts have been observed, between $H_{-1} 1^{\prime}$ and H-6pro-S,6pro-R, respectively. The experimental ROEs for these contacts are more intense than those calculated on the basis of any distance matrix, as is visualized in Fig. 8. In order to estimate the distance involved, the intensities at $\tau_{\mathrm{m}}=200 \mathrm{~ms}$ have been translated into distances by the use of eq 2 , affording distances of 3.0 and $2.8 \AA$ for $\mathrm{H}-1^{\prime}$ to $\mathrm{H}-6$ pro- $S, 6$ pro- $R$, respectively. The distances in $\mathbf{D}_{\mathrm{A}}$ are closest, being 3.5 and $3.3 \AA$, respectively. The matrix $\mathbf{D}_{\mathrm{B}}$ yields the worst results with distances of 4.6 and $4.5 \AA$, respectively. It is clear that models $\mathbf{D}_{\mathrm{A}}, \mathbf{D}_{\mathrm{C}}$, and $\mathbf{D}_{\mathrm{D}}$ (pure 1) have a better agreement with the experimental ROE than $D_{B}$ (pure 3). Mixture of conformations from 1 and 3 cannot therefore possibly give a better result than the average conformation of region 1 . It is therefore unlikely that conformations in region 3 contribute significantly, because this would need compensation by conformations in region 2 with their long $\mathrm{H}-1^{\prime} \cdots \mathrm{H}-3,4,5$ distances. The latter, however, is not regarded as particularly stable.

Model $D_{A}$ has a too short average $H-1^{\prime} \cdots H-4$ distance $(1.99 \AA ̊ 丿)$ in view of the experimental $R O E$ intensity. Models $\mathbf{D}_{C}$ and $\mathbf{D}_{\mathrm{D}}$, which are obtained from a longer simulation, have a larger $H-1^{\prime} \cdots H-4$ distance $(2.09 \AA)$ and therefore give a significantly better result. It appears that a long simulation is needed to sample the broad region 1 properly. Nevertheless, the experiment suggests an even larger distance. Inspection of the trajectory displayed in Fig. 9 of the $\mathrm{H}-1^{\prime} \cdots \mathrm{H}-4$ distance against the torsional angle $\phi$ during the 500-ps simulation in region 1 reveals that larger $\mathrm{H}-1^{\prime} \cdots \mathrm{H}-4$ distances are obtained at more positive $\phi$ values. The correlation between the $\mathrm{H}-1^{\prime} \cdots \mathrm{H}-4$ distance and $\psi$ is less distinct. Therefore, a better agreement with the experimental ROE can be obtained when the balance of $(\phi, \psi)$ is shifted to somewhat more positive $\phi$ values. The general conclusion is therefore that methyl $\beta$-cellobioside resides in region 1 and has considerable conformational flexibility, especially with respect to $\phi$.

It is estimated from CROSREL data that the observed $\mathrm{H}-\mathbf{1}^{\prime}-\mathrm{H}-3,4,5$ cross-peak corresponds to a $\mathrm{H}-1^{\prime} \cdots \mathrm{H}-4$ distance of $2.22 \AA$. The initial rate analysis yielded two estimates for the $\mathrm{H}-\mathbf{1}^{\prime} \cdots \mathrm{H}-4$ distance, based on the $\mathrm{H}-1-\mathrm{H}-3,5$ contacts or $\mathrm{H}-6$ pro-S $-\mathrm{H}-6$ pro- $R$ contacts as references, that were somewhat shorter. It was 


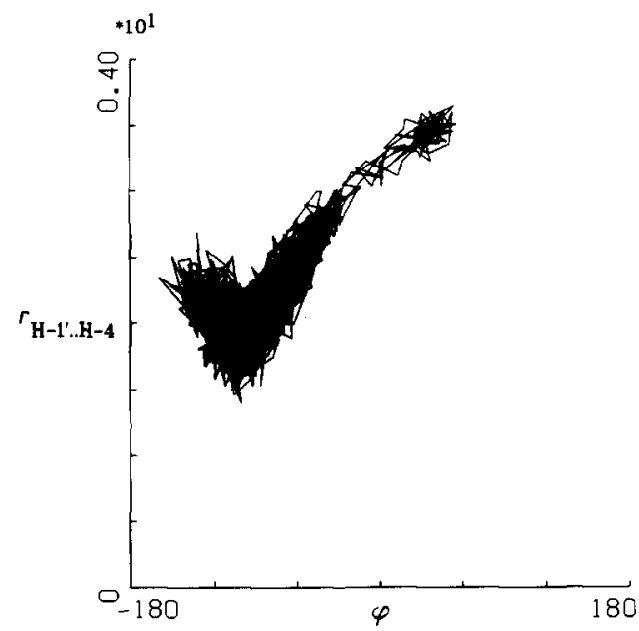

Fig. 9. Trajectory of the length of the H-1 $-\mathrm{H}-4$ vector (in $\AA$ ) plotted against the torsional angle $\phi$ (in degrees) during 500-ps MD simulation.

expected that the mobility of the H-6 atoms is higher than that of the ring protons. This is indeed what is reflected in the individual $\tau_{c}$ values listed in Table I. Since the initial rate approach implicity assumes isotropic tumbling of the molecule, that reference should be used which resembles the unknown proton pair best with respect to the overall mobility. However, on the basis of the initial rate data alone, no decision could be made regarding which reference should be preferred, and therefore the average value was taken. When the individual $\tau_{c}$ values of the $\mathrm{H}-1^{\prime}-\mathrm{H}-4$ vector (39.5 ps) are compared with those of $\mathrm{H}-1^{\prime}-\mathrm{H}-3,5$ (on average, 48.5 ps) and H-6pro-S-H-6pro-R (on average, $30.2 \mathrm{ps}$ ), this approach seems to be valid.

The differences between the distances obtained by the full matrix approach and by the initial rate approach are surprisingly small. Nevertheless, a preference exists for the former, since it allows a full correction for the offset dependence of ROESY peak intensities, and incorporates the effects of direct HOHAHA transfer in a straightforward manner. Also with respect to the molecular tumbling, the CROSREL method is more flexible than the initial rate method, which implicitly assumes isotropic motion. Concerning the relaxation process, the potential problems for the initial rate approach can be reduced to the NOE type contribution to this process. When this type of relaxation is significant, it influences the peak intensities such that a simple correction for the offset effect is not possible. Since the NOE contribution is insignificant for methyl $\beta$-cellobioside measured at 500 $\mathrm{MHz}$, the initial rate method results presented here do not suffer from this problem. A prerequisite for the HOHAHA correction is knowledge of the initial magnetization, which can be deduced from all (including diagonal) peak intensities. Although these are known for the small molecule methyl $\beta$-cellobioside, this will not generally be so for larger and more complex molecules. An additional advantage of the CROSREL method is that NMR spectra acquired with longer 
mixing times and high signal-to-noise ratios can be used, whereas, for the initial rate approach, experiments with shorter mixing times and lower signal-to-noise ratios are more important. The latter experiments also suffer more from the remains of the oscillatory character of HOHAHA transfer.

\section{CONCLUSIONS}

The structure of methyl $\beta$-cellobioside in aqueous solution is established to be primarily in one minimum energy region of the glycosidic torsional angles $\phi$ and $\psi$. This region (1) corresponds to the conformations found in the crystal structures of $\beta$-cellobiose, methyl $\beta$-cellobioside, and cellulose I and II. In the crystal structures, the HO-3 to O-5' hydrogen bond exists, which is not the case in solution, as was established previously ${ }^{18}$. It is particularly interesting that, without this hydrogen bond, conformations in region 1 are still dominant, although a large spread in $(\phi, \psi)$ is found. On extrapolation, this has some implications for the behaviour of cellulose molecules in solution. The molecule is confined to a single region in $(\phi, \psi)$ space and will therefore show considerable rigidity that is not due to an intramolecular hydrogen bond as is often assumed.

\section{ACKNOWLEDGMENTS}

The authors thank Professor Dr. W.F. van Gunsteren for the use of the GROMOS program package. This work was supported by the Netherlands Program for Innovation Oriented Carbohydrate Research (IOP-k) with financial aid of the Ministry of Economic Affairs and the Ministry of Agriculture, Nature management, and Fisheries.

\section{REFERENCES}

1 K.H. Gardner and J. Blackwell, Biopolymers, 13 (1974) 1975-2001.

2 R.H. Marchessault and P.R. Sundararajan, in G.O. Aspinall (Ed.), The Polysaccharides, Vol. 2, Academic Press, London, 1983, Chapter 2.

3 K.J. Kolpack and J. Blackwell, Macromolecules, 9 (1976) 273-278.

4 I. Simon, L. Glasser, H.A. Scheraga, and R. St. John Manley, Macromolecules, 21 (1988) 990-998.

5 I. Simon, H.A. Scheraga, and R. St. John Manley, Macromolecules, 21 (1988) 983-990.

6 A.A. Bothner-By, R.L. Stephens, J.-M. Lee, C.D. Warren, and R.W. Jeanloz, J. Am. Chem. Soc., 106 (1984) 811-813.

7 B.R. Leeflang and L.M.J. Kroon-Batenburg, J. Biomol. NMR, 2 (1992) 495-518.

8 A. Bax, J. Magn. Reson., 77 (1988) 134-147.

9 R. Boelens, T.M.G. Koning, G.A. van der Marel, J.H. van Boom, and R. Kaptein, J. Magn. Reson., 82 (1989) 290-309.

10 F. Smith and J.W. Van Cleve, J. Am. Chem. Soc., 74 (1952) 1912-1913.

11 K.F. Kuhlmann, D.M. Grant, and R.K. Harris, J. Chem. Phys., 57 (1970) 3439-3448.

12 A. Bax and D.G. Davis, J. Magn. Reson., 63 (1985) 207-213.

13 G. Urobney, A. Pines, S. Sinton, D. Weitekamp, and D. Warren, Faraday Div. Chem. Soc. Symp., 13 (1979) 49-55.

14 G. Bodenhausen, R.L. Vold, and R.R. Vold, J. Magn. Reson., 37 (1980) 93. 
15 D. Marion and K. Wüthrich, Biochem. Biophys. Res. Commun., 113 (1983) 967-974.

16 W.F. van Gunsteren, GROMOS, Groningen Molecular Simulation Package, University of Groningen, The Netherlands, 1987.

17 J. Koehler, W. Saenger, and W.F. van Gunsteren, Eur. Biophys. J., 15 (1987) 197-210.

18 B.R. Leeflang, L.M.J. Kroon-Batenburg, B.P. van Eijck, J. Kroon, and J.F.G. Vliegenthart, Carbohydr. Res., 230 (1992) 41-61.

19 J.M. Scarsedale, R.K. Yu, and J.H. Prestegard, J. Am. Chem. Soc., 108 (1986) 678-684.

20 A. Kumar, G. Wagner, R.R. Frnst, and K. Wüthrich, J. Am. Chem. Soc., 103 (1981) 3654-3658.

21 G. Lipari and A. Szabo, J. Am. Chem. Soc., 104 (1982) 4546-4559.

22 A.D. French, Carbohydr. Res., 188 (1989) 206-211.

23 R.W.W. Hooft, J.A. Kanters, and J. Kroon, J. Comput. Chem., 12 (1991) 943-947.

24 B. Henrissat, S. Perez, I. Tvaroska, and W.T. Winter, ACS Symp. Ser., 340 (1987) Chapter 3.

25 S. Melberg and K. Rasmussen, Carbohydr. Res., 71 (1979) 25-34.

26 S.S.C. Chu and G.A. Jeffrey, Acta Crystallogr., Sect. B, 24 (1968) 830-838.

27 J.T. Ham and D.G. Williams, Acta Crystallogr., Sect. B, 26 (1970) 1373-1383.

28 B.R. Leeflang, Ph.D. Thesis, Conformational analysis of oligosaccharides, Utrecht University, 1991, Chapter 1.

29 A. Sarko, C.-H. Chen, B.J. Hardy, and F. Tanaka, ACS Symp. Ser., 430 (1990) Chapter 21.

30 L.M.J. Kroon-Batenburg and J. Kroon, Biopolymers, 29 (1990) 1243-1248. 